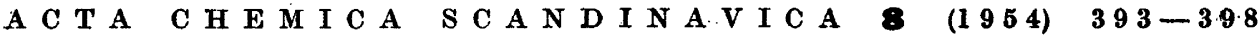

\title{
On the Quantitative Determination of Solids by Means of Infrared Spectroscopy. Determination of Benzylpenicillin in Crystalline Sodium Salts
}

J. B U UR J E NSE N

Leo Pharmaceutical Products, Copenhagen, Denmark

M M. Stimson and M. T. O'Donnel ${ }^{1}$ in 1952 published an article on the preparation of discs of potassium bromide able to transmit infrared radiation by the compression at high pressure of finely ground potassium bromide. According to this method it should be possible to record the infrared spectrum of a substance in the solid state by mixing the substance with potassium bromide. Similar and more comprehensive experiments have been made independently by the above authors by U. Schiedt and $\mathrm{H}$. Rheinwein ${ }^{2}$.

Both the above mentioned articles deal only with qualitative evaluations of the spectra, but Schiedt and Rheinwein mention that the spectra may also be used for quantitative determinations. As the number of solvents which are suitable for absorption measurements in the infrared range is extremely limited $^{3}$, it is evident that every improvement of the experimental technique in connection with the recording of spectra of solids must be considered an important advance. The methods hitherto used, according to which the absorption measured is either that of a suspension of the substance in liquid paraffin or that of a thin film of the substance on a sodium chloride window, are not readily reproducible and consequently more suitable for qualitative determination.

The new method seemed to involve many advantages and was therefore subjected to a close study. Both the authors quoted employ a compression device which allows for the evacuation of the potassium bromide powder before and during the pressing. By means of such a device Schiedt and Rheinwein are able to prepare discs with a transmittancy of $90 \%$ in the wavelength range $7-15 \mu$. For the purpose of the present work it was, unfortunately, not possible to work under similar conditions, and a simpler method was tried. A pressing device, as illustrated in Fig. 1, was made from steel and hardened to $60^{\circ}$ Rockwell " $C$ " hardness. The die was surrounded by a ring of mild steel to prevent accidents in case the die should burst. A hydraulic press was used to establish the high pressure, and after the pressing the resulting potassium bro-

Acta Chem. Scand. 8 (1954) No. 3 
mide disc was fitted in a suitable holder in the spectrograph for the purpose of the measurements. The spectrograph used was a Beckman I R. II spectrograph with sodium chloride prism and recorder. This simple procedure has also been tried by Schiedt and Rheinwein, who consider it to be unsatisfactory because the discs have a cloudy appearance, presumably on account of air inclusions.

In spite of the cloudy appearance the transmittancy of such dises is not less than $70-80 \%$ within the range $7-15 \mu$.

As the intensity losses are due to reflection and scattering, it should be expected that the measured transmittancy would be dependent on the orientation of the disc in the spectrograph, an assumption which has been confirmed by experiments. Experiments proved, however, that the transmittancy for a certain orientation of the disc is constant within the range 7-15 $\mu$. From this it will be seen that it will be impossible to prepare blank discs for quantitative measurements, while it will be possible to use the base-line technique proposed by Wright 4 , thus eliminating the necessity of a blank value.

By means of the above described simple pressing technique it has proved possible to verify most of Schiedt and Rheinwein's results. Out of several alkali halogenides potassium bromide thus appeared to be the most suitable. By way of example it may be mentioned that the transmittancies of two discs of sodium chloride and potassium bromide - prepared under identical conditions - were 30-40\% and 65-75\%, respectively. It was also found that fine grinding and effective drying are factors which increase the transmittancy of the discs. For the subsequent experiments the substance used was micronized potassium bromide a.g., Merck, which after the micronizing was dried for 24 hours at $110^{\circ} \mathrm{C}$.

The aim of the first experiments was to investigate the influence of the pressure used for preparing the discs on the transmittancy. Discs of $0.5 \mathrm{~g}$ with a diameter of $16 \mathrm{~mm}$ were prepared at different pressures, ranging from 25 tons to 45 tons. The period through which the pressure acted was in all cases 2 minutes. On examination it proved impossible to measure any significant differences in the transmittancy of the discs.

The aim of the following experiments was to investigate whether the duration of the compression had any influence on the transmittancy. Discs were therefore prepared at a pressure of 25 tons and with the same weight and diameter as mentioned above, but the period of compression varied from $15 \mathrm{se}-$ conds to 10 minutes. A period of 1 minute proved to be sufficient to produce maximum transmittancy, for which reason a pressure of 25 tons and a period of compression of 1 minute were subsequently considered to produce optimum conditions.

The dises resulting from this procedure have - as mentioned above - a transmittancy of $70-80 \%$ within the range $7-15 \mu$.

\section{DETERMINATION OF BENZYLPENICILLIN}

The determination of benzylpenicillin in crystalline sodium salts is an example of the application of this method for a quantitative purpose. Two modifications of a method for the determination of benzylpenicillin in crystalline salts by means of infrared spectroscopy have been described in the litera- 

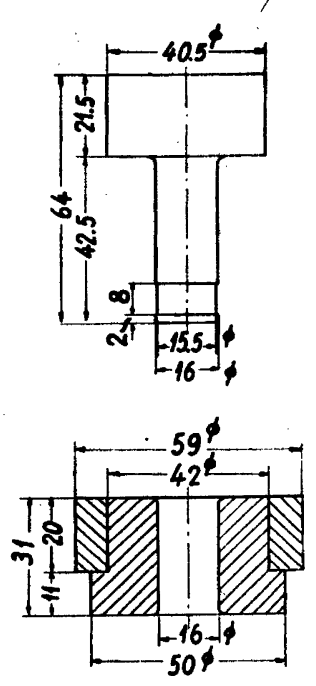

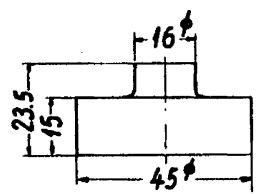

Fig. 1. Pressing device.

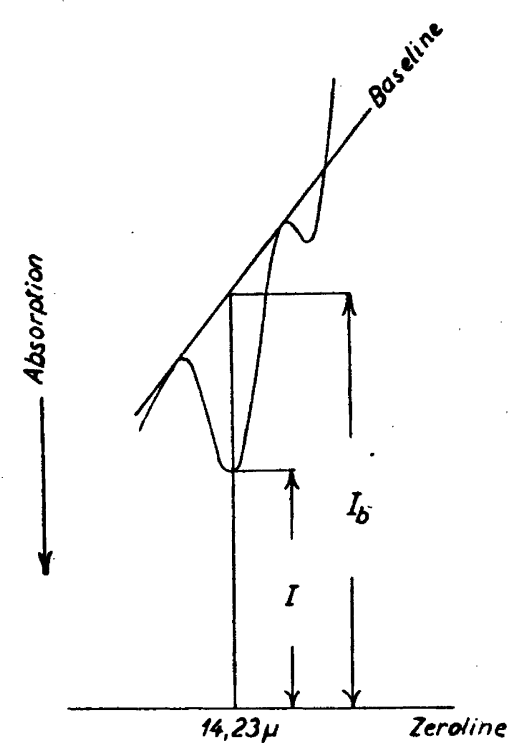

Fig. 2. Determination of base-line extinction Base-line extinction $=\log I_{b} / I$.

ture by Barnes and coworkers ${ }^{5}$ and E. A. Garlock jr. and D. C. Grove ${ }^{6}$, respectively.

Both modifications are based on measurements of suspensions of penicillin in liquid paraffin, and both utilize the absorption band with maximum at $14.23 \mu$ which is characteristic of benzylpenicillin. The difference between the procedures lies in the different ways in which the thickness of the layer is eliminated. Barnes et al. apply the internal standard technique using $\mathbf{D}, \mathbf{L}_{-}$alanine as internal standard. This procedure, however, requires that a constant ratio always be maintained between the amount of substance weighed out for the analysis and the amount of D,L-alanine used. Garlock and Grove use a cell which ensures constant thickness of the layer. None of the methods are quite satisfactory, and the author's own experiments show that analyses according to both of them must be made with the greatest care and by an experienced analyst to give reliable results.

When applying the new technique, the thickness of the layer may be neglected as it is possible to weigh the finished disc.

Lambert-Beer's law applies in the following form:

$$
\log \frac{I_{0}}{I}=E . c . l
$$

Acta Chem. Scand. 8 (1954) No. 3 
where $I_{0}$ is the incident energy, $I$ the energy which has passed through the disc, while $E$ is a constant, $l$ the thickness of the layer and $c$ the concentration.

If $F$ denotes the area of the disc and $a$ is an expression of the quantity of substance uniformly distributed throughout the disc, the following equation will apply:

$$
c=\frac{a}{F \cdot l}
$$

Substituting this in $(\mathbf{I})$, we obtain

$$
\log \frac{I_{0}}{I}=\frac{E \cdot a}{\mathrm{~F}}=K \cdot a
$$

As the press used is always the same, $K$ is a constant.

If a mixture of sodium benzylpenicillin and potassium bromide of known composition is prepared, it should be possible to obtain a straight line relationship between the weight of the discs prepared from this mixture and the extinction, that is, a standard curve.

It has already been mentioned that it is impossible to prepare a blank disc, for which reason the extinction must be replaced by the base-line extinction. The meaning of this term appears in Fig. 2 which shows the infrared spectrum of sodium benzylpenicillin in the range about $14.2 \mu$ as produced by the recorder of the spectrograph.

For the present experiments a crystalline sodium salt was used which fulfilled the requirements of the Danish Pharmacopoea as to identity and purity ${ }^{7}$. The content of benzylpenicillin was found to be $98 \%$ according to the N-ethylpiperidine precipitation method. $249 \mathrm{mg}$ of this salt was ground carefully in a mortar and mixed with $50.00 \mathrm{~g}$ micronized potassium bromide, a. g. Merck. Samples of $0.2-1.0 \mathrm{~g}$ of this mixture were now subjected to a pressure of 25 tons for 1 minute. The absorption of the resulting discs was measured at once in the range 13.8-14.8 $\mu$; slit and amplification being chosen once and for all so as to cause the recorder to give full deflection at $13.8 \mu$. After the recording of the spectrum, the disc was weighed on an analytical balance to obtain an expression of the quantity of substance involved.

Fig. 3. illustrates the results of a series of measurements. The base-line extinction is in this case plotted against the weight of the disc. It can be seen from this graph that the relationship is only linear up to about $0.6 \mathrm{~g}$ of mixture, after this value the curve flattens out considerably. The reason is that the absorption exhibited by the heaviest discs is too great to allow for a reasonably accurate determination of the base-line extinction. Experiments now showed that by using a mixture containing only half the amount of sodium benzylpenicillin $(0.25 \%)$ it was possible to obtain the same standard curve when applying discs of twice the original weight.

The following method was used for a series of analyses: $0.0500 \mathrm{~g}$ of substance was finely ground and mixed thoroughly with $10.00 \mathrm{~g}$ of micronized potassium bromide, a. g. Merck. When applying a micro balance, it is possible to use quantities only one tenth of those mentioned. From this mixture discs with a weight of $0.3-0.5 \mathrm{~g}$ were prepared as described above. The spectrum was recorded under standard conditions, and the base-line extinction at $14.23 \mu$ 


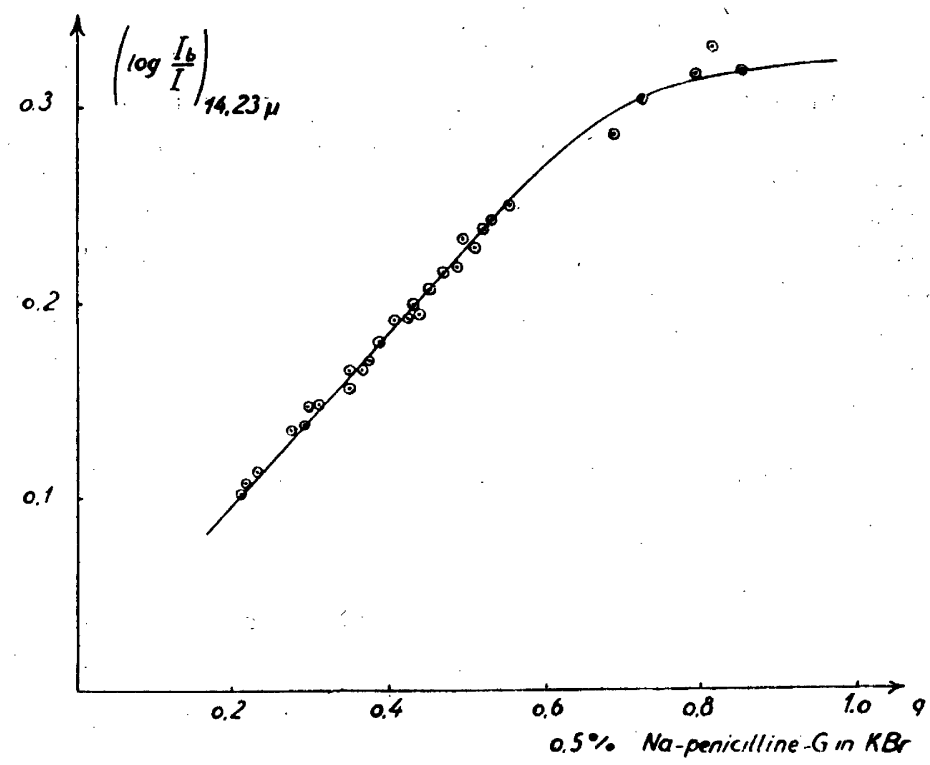

Fig. 3. Standard curve for the determination of benzylpenicillin.

was determined. When the weight of the disc has been determined, the necessary data are available for the calculation by means of the standard curve of the content of benzylpenicillin. A series of determinations made on the same salt sample (Table 1) illustrates the reproducibility of the method.

Table 1. Repeated determinations on the same salt sample.

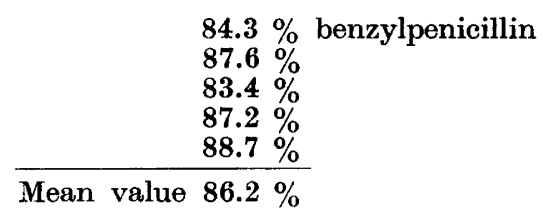

Four salt samples of different origin were analysed, according to the precipitation method described in the Danish Pharmacopoea and also according to the method described in the present work. A comparison of the results is given in Table 2.

Table 2

Salt sample no.

N. E. P.

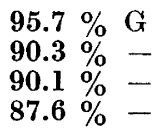

1

2

4

1
2
3
4

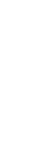

$$
\text { no. }
$$

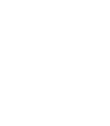

\footnotetext{
Acta Chem. Scand. 8 (1954) No. 3
} 


\section{COMMENTS}

Barnes et al..$^{5}$ have given a thorough discussion of the sources of error which may be the result of absorption bands near $14.2 \mu$ due to interfering substances. It is consequently necessary to examine the whole spectrum qualitatively in order to exclude the possibility of interference from impurities.

The grinding of the sample to be analyzed has been performed in a mortar before the admixture of potassium bromide. Since the absorption is to some extent dependent on the particle size ${ }^{8}$, it is essential to perform the grinding carefully so as to obtain uniform particle size. Schiedt and Rheinwein suggest solution of the sample in alcohol followed by admixture of a known quantity of potassium bromide and evaporation in vacuum of the alcohol. This method may perhaps more readily produce a uniform mixture of the two substances, but it is doubtful whether the particle size obtained is uniform from time to time. As sodium penicillin is rather labile, this method has not been taken into consideration for the purpose of the present work.

In comparison with the usual $\mathrm{N}$-ethylpiperidine method the method suggested in the above is distinguished by being rapid and just as reliable, the analytical error being about $3 \%$. In comparison with previously used methods based on infrared spectroscopy the method is more readily reproducible, because the elimination of the thickness of the layer does not require special measures.

\section{SUMMARY}

Compression at high pressure of finely ground potassium bromide results in discs with $70-80 \%$ transmittancy in the wavelength range $7-15 \mu$. The addition of substances to the bromide enables recordings of the infrared spectra of such substances in the solid state. The spectra recorded may be utilized for quantitative determinations when applying the base-line technique proposed by Wright. On this basis a method for the determination of benzylpenicillin in crystalline sodium salts has been developed and described in the present work, the measurements being referred to the specific maximum at $14.23 \mu$ which is characteristic of benzylpenicillin.

\section{REFERENCES}

1. Stimson, M. M. and O'Donnel, M. J. J. Am. Chem. Soc. 74 (1952) 1805.

2. Schiedt, U. and Rheinwein, H. Z. Naturforsch. 7b (1952) 270.

3. Torkinton, P. and Thompson, H. W. Trans. Faraday Soc. 41 (1945) 184.

4. Wright, N. Ind. Eng. Chem. Anal. Ed. 13 (1941) 1.

5. Barnes, R. B., Gore, R. C., Williams, E. F., Linsley, S. G. and Petersen, E. M. Anal. Chem. 19 (1947) 620.

6. Garlock, E. A. jr. and Grove, D. C. J. Am. Pharm. Assoc. Sci. Ed. 37 (1948) 409.

7. Pharmacopoea Danica IX add., 1952, pag. 80.

8. Henry, R. C. J. Opt. Soc. Amer. 38 (1948) 775.

Received December 18, 1953. 